\title{
Imaging through turbulence with a quadrature-phase optical interferometer
}

\author{
Brian Kern, Paul E. Dimotakis, Chris Martin, Daniel B. Lang, and Rachel N. Thessin
}

\begin{abstract}
We present an improved technique for imaging through turbulence at visible wavelengths using a rotation shearing pupil-plane interferometer, intended for astronomical and terrestrial imaging applications. While previous astronomical rotation shearing interferometers have made only visibility modulus measurements, this interferometer makes four simultaneous measurements on each interferometric baseline, with phase differences of $\pi / 2$ between each measurement, allowing complex visibility measurements (modulus and phase) across the entire input pupil in a single exposure. This technique offers excellent wavefront resolution, allowing operation at visible wavelengths on large apertures, is potentially immune to amplitude fluctuations (scintillation), and may offer superior calibration capabilities to other imaging techniques. The interferometer has been tested in the laboratory under weakly aberrating conditions and at Palomar Observatory under ordinary astronomical observing conditions. This research is based partly on observations obtained at the Hale Telescope. (C) 2005 Optical Society of America
\end{abstract}

OCIS codes: $120.3180,010.1080,350.1260$.

\section{Introduction}

Propagation through turbulence degrades the angular resolution that can be obtained by direct imaging. ${ }^{1}$ Under excellent astronomical conditions, the angular resolution at visible wavelengths is limited to the neighborhood of 0.5 arc sec, far worse than a comparable diffraction limit, $\lambda / D=0.010$ arc sec (for $\lambda=0.5 \mu \mathrm{m}, D=10 \mathrm{~m}$ ). Several techniques have been developed to obtain visible and near-IR images through turbulence, with resolution approaching the diffraction limit. These techniques include adaptive optics (AO), long-baseline interferometry, speckle imaging, nonredundant masking, blind deconvolution, and pupil-plane shearing interferometry.

The past decade has seen extraordinary progress in the field of visible and near-IR AO on large groundbased telescopes. ${ }^{2}$ AO systems obtain a reference wave-front phase map using a natural or artificial guide star, and apply phase corrections to the incoming light with deformable mirrors or other real-time phase modulators. Deformable mirrors have a finite number of actuators, $N$, which sets an upper limit to

The authors are with the California Institute of Technology, 1200 East California Boulevard, Pasadena, California. B. Kern's e-mail address is bdk@srl.caltech.edu.

Received 12 November 2004; revised manuscript received 21 May 2005; accepted 24 May 2005.

0003-6935/05/347424- $15 \$ 15.00 / 0$

(C) 2005 Optical Society of America the ratio of the telescope diameter, $D$, to the phase coherence length, ${ }^{3} r_{0}$, of approximately $D / r_{0}=N^{1 / 2}$. Deformable mirrors with hundreds to approximately a thousand actuators enable AO systems to operate at good astronomical sites in near-IR wavelengths with $10 \mathrm{~m}$ apertures, ${ }^{4}$ or visible wavelengths with $3.6 \mathrm{~m}$ apertures. ${ }^{5}$

A handful of long-baseline interferometers, which combine light from separate apertures, are capable of producing images with resolution $\lambda / B$, where $B$ is the baseline (separation) between apertures. ${ }^{6}$ Individual apertures must either have diameters comparable to $r_{0}$, or else have $\mathrm{AO}$ systems operating on each aperture to produce flat wavefronts for interference. The ability of an interferometer to form images of complicated fields is determined by the number of baselines over which interference is measured, and whether complex visibilities or only visibility moduli are measured. Long-baseline interferometers tend to be quite complex (and expensive), with the complexity increasing for systems capable of producing high-quality images. Imaging long-baseline interferometers have only recently begun to explore angular resolutions that are unobservable using single (large) apertures. The sensitivity of such systems depends primarily on the individual aperture sizes and the wavelength.

Alternative passive methods (methods with no actively moving components) for obtaining diffractionlimited images from single apertures have been developed over the past three decades. These meth- 
ods include speckle imaging, ${ }^{7}$ nonredundant masking, ${ }^{8}$ blind deconvolution, ${ }^{9}$ and pupil-plane shearing interferometry. ${ }^{10-13}$ In their simplest forms, these techniques use short exposures to freeze turbulent aberrations, and obtain images by a variety of postprocessing techniques. In speckle imaging, shortexposure focal-plane images retain some information content (greatly attenuated) at high angular frequencies, which can be restored by calibrating the Fourier transforms of the images using concurrent observations of unresolved (pointlike) sources. In nonredundant masking, the input pupil is masked so that only a small number of subapertures transmit light; their interference patterns are observed in the focal plane. Light interfering from each pair of subapertures can be separately identified in Fourier transforms, from which closure phase relationships can be determined. Closure phases are unaffected by turbulent aberrations, and allow images to be reconstructed, subject to some uncertainties regarding symmetries and uniqueness. Blind deconvolution is similar to speckle imaging but requires essentially no calibration. It is subject to large systematic uncertainties and uniqueness issues.

The most efficient form of pupil-plane shearing interferometry, when attempting to reconstruct images using a large number of baselines, is rotation shearing interferometry. A rotation shearing interferometer combines two identical copies of the input pupil, rotated with respect to one another, and records the resulting interferograms (in the pupil plane) on a detector. The rotation shear results in a large number of independent baselines being observed simultaneously. The first rotation shearing interferometer to have been used for astronomy was designed ${ }^{10}$ and operated $^{14}$ in the early 1970 's. This visible-light interferometer obtained visibility amplitude measurements but no phase information. An interferometer of the same design, with the addition of a phase plate to compensate for polarization-dependent phase shifts and improve visibility modulus measurements, was used to obtain high-resolution images of Betelgeuse. ${ }^{11,15}$ An infrared rotation shearing interferometer was constructed that used fringe scanning to attempt to measure the visibility phase from a time series of exposures, ${ }^{12}$ but the magnitude limit was quite bright and the atmosphere changed on time scales faster than the fringe-scanning exposures. An additional pupil-plane interferometer produced preliminary fringes but does not appear to have been pursued further. ${ }^{13}$ The design and construction of other rotation shearing interferometers have been discussed in the literature, but astronomical observations using these interferometers have not been published. To date, the use of rotation shearing interferometers in astronomy has been largely limited to observations of Betelgeuse.

Rotation shearing interferometry is also used in a Fizeau arrangement, where the detector lies in the telescope focal plane rather than the pupil plane. This arrangement may be used to provide nulling of on-axis light, allowing searches for exozodiacal disks and plan- ets around nearby stars, for example. ${ }^{16}$ This interferometer arrangement, while optically similar to pupil-plane rotation shearing interferometry, operates in a fundamentally different manner, and provides no capability for improving image resolution beyond the seeing limit. Focal plane rotation shearing interferometry will not be discussed further in this paper.

We have developed a technique of rotation shearing interferometry that obtains phase information using an instantaneous phase-shifting interferometer optical arrangement. This arrangement measures phase in four separate interferograms, ideally with instrumental phase shifts of $\pi / 2 \operatorname{rad}\left(90^{\circ}\right)$ between each (i.e., phase shifts of $0, \pi / 2, \pi$, and $3 \pi / 2 \mathrm{rad}$ ), making this interferometer a quadrature-phase interferometer (QPI). This technique allows instantaneous determination of the complex visibility (modulus and phase) using only a single exposure that, after removing turbulent phase aberrations, allows image reconstruction at the diffraction limit of the system, subject to signal-to-noise limitations.

Because this is a pupil-plane interferometry technique, the interferograms recorded on the detector are superposed, rotated images of the input pupil. The number of pixels on the detector then determines the wave-front spatial resolution. We have developed a high-speed charge-coupled device (CCD) camera to operate with the QPI, which offers a relatively large number of pixels $(1024 \times 1024)$ and short read times (5-10 ms full-frame readout). The spatial resolution in the input pupil is then approximately $D / 512$, where $D$ is the input pupil diameter. Generally speaking, this allows operation up to $D / r_{0} \sim 100$, i.e., finer than the scales at which typical AO systems can make phase corrections (as limited by number of actuators).

This paper describes the design, testing, and preliminary operation of the QPI. We present data from both laboratory and astronomical observations.

\section{Design}

\section{A. Interferometric Imaging}

The goal of any imaging technique, direct or interferometric, is to estimate the two-dimensional object brightness map, $B(\alpha, \beta)$, as a function of the angles $\alpha$ and $\beta$. The fundamental connection between interferometry and imaging is the van Cittert-Zernike the orem, ${ }^{17,18}$ which states that the Fourier transform of the object brightness map, $B(\alpha, \beta)$, is the mutual coherence function, $\gamma(u, v)$,

$$
\mathscr{F}[B(\alpha, \beta)]=\gamma(u, v) .
$$

The mutual coherence function is also known as the complex visibility,

$$
\gamma(u, v)=V(u, v) \exp \{i \phi(u, v)\},
$$

where $V(u, v) \in[0,1]$ is the visibility modulus, and $\phi(u, v)$ is the visibility phase (both $V$ and $\phi$ are real valued). Visible-light interferometers measure intensities modulated by the complex visibility, 


$$
\begin{aligned}
I(u, v) / I_{0} & =1+\Re\left\{\gamma_{\text {meas }}(u, v)\right\}, \\
& =1+V_{\text {meas }}(u, v) \cos \left[\phi_{\text {meas }}(u, v)\right] .
\end{aligned}
$$

The measured visibility modulus, $V_{\text {meas }}$, and visibility phase, $\phi_{\text {meas }}$, contain contributions from various instrumental and aberrating conditions, as described below. Forming an image with an interferometer can then be described as obtaining $\gamma_{\text {meas }}$ over a range of $(u, v)$ points, estimating $\gamma$ from $\gamma_{\text {meas }}$, and obtaining $B$ through Eq. (1). In all cases considered here, it is assumed that the emission from the object is mutually incoherent at the emission location, which is a necessary condition for use of the van Cittert-Zernike theorem.

The coordinates $u$ and $v$ in the preceding equations are angular frequencies, which arise in the Fourier transform from angles $\alpha$ and $\beta$ of the object brightness map $B(\alpha, \beta)$. Denoting spatial coordinates of the input pupil by $\xi$ and $\eta$, the interference of light from points $\left(\xi_{A}, \eta_{A}\right)$ and $\left(\xi_{B}, \eta_{B}\right)$ corresponds to an interference baseline $\left(\xi_{B}-\xi_{A}, \eta_{B}-\eta_{A}\right)$, and an angular frequency coordinate

$$
(u, v)=\left[\left(\xi_{\mathrm{B}}-\xi_{\mathrm{A}}\right) / \lambda, \quad\left(\eta_{\mathrm{B}}-\eta_{\mathrm{A}}\right) / \lambda\right],
$$

for quasi-monochromatic light at a wavelength $\lambda$. The intensity observed for the interference of the two points $\left(\xi_{\mathrm{A}}, \eta_{\mathrm{A}}\right)$ and $\left(\xi_{\mathrm{B}}, \eta_{\mathrm{B}}\right)$ from the input pupil is given by Eq. (3), using Eqs. (1), (2), and (4). A schematic representation of interference of two points in the input pupil is shown in Fig. 1.

The object brightness map, $B(\alpha, \beta)$, is always real valued, which makes the complex visibility, $\gamma(u, v)$, Hermitian, so that

$$
\begin{gathered}
\gamma(u, v)=\gamma^{*}(-u,-v), \\
V(u, v)=V(-u,-v), \\
\phi(u, v)=-\phi(-u,-v) .
\end{gathered}
$$

One consequence of this is that $\gamma(u, v)$ is fully defined if it is measured over only half of the $(u, v)$ plane, e.g., for $u>0$.

\section{B. Rotation Shearing Interferometry}

Rotation shearing interferometers interfere light by making two copies of the input pupil, rotating the two copies with respect to one another, and recombining them to form an interferogram. The QPI is a $180 \mathrm{deg}$ rotation shearing interferometer, meaning that the two copies of the input pupil are sent into two arms of the interferometer that rotate the input pupil copies by $180 \mathrm{deg}$ with respect to one another before being recombined. The interferometer can also introduce an instrumental path-length difference (and therefore an instrumental phase) between the optical paths of light through the two arms. A schematic representation of this rotation shearing operation is shown in Fig. 2, and the mirror configuration used to

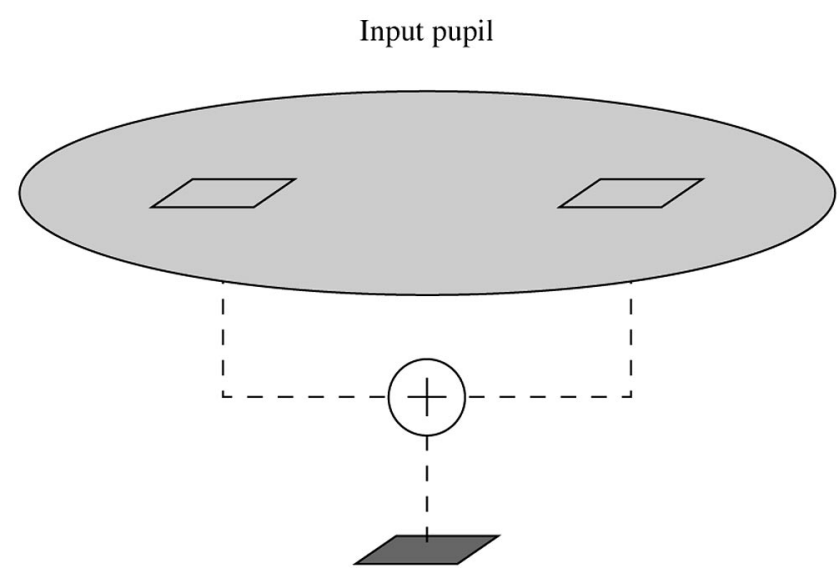

Fig. 1. Schematic description of interference between two points in the input pupil. Light from two subapertures of the input pupil is superposed (with the superposition shown as $\oplus$ ). The output intensity is modulated by the complex visibility, evaluated at a frequency defined by the separation between the two subapertures. In the case of a rotation shearing interferometer, the subapertures are defined by individual pixels on the detector, and all points in the input pupil are simultaneously interfered pairwise to form a two-dimensional interferogram.

accomplish the rotation shear is shown in Fig. 3. The QPI is arranged in a Mach-Zehnder geometry, so that two full-pupil interferograms are output, as opposed to a Michelson geometry, in which one interferogram is reflected back toward the input pupil. Sample interferograms are shown in Fig. 4. The two interferograms differ in the instrumental phase, as described below.

By virtue of the rotation shear, each point in the input pupil is combined with its diametrically opposed point. Locations in the interferograms define a third coordinate system, designated by $(x, y)$, which correspond to detector pixels and are distinct from the input pupil coordinates, $(\xi, \eta)$, and the angular frequencies $(u, v)$. Because each interferogram is the superposition of two copies of the input pupil, the interferogram $(x, y)$ coordinate systems have the same physical dimensions as the input pupil $(\xi, \eta)$ coordinate system, with two rotations applied. There are two $(x, y)$ coordinate systems, one for each interferogram, with origins at the center of each full-pupil interferogram. A point $(x, y)$ in an interferogram records the interference between points $\left(\xi_{\mathrm{A}}, \eta_{\mathrm{A}}\right)$ $=(y,-x)$ and $\left(\xi_{\mathrm{B}}, \eta_{\mathrm{B}}\right)=(-y, x)$, corresponding to an angular frequency of

$$
(u, v)=(2 y / \lambda,-2 x / \lambda) .
$$

A comparable $(x, y)$ point exists in each of the two full-pupil interferograms, each corresponding to interference of the same two points in the input pupil, and therefore to the same $(u, v)$ location.

\section{Measured Visibility Phase Components}

The measured visibility phase contains contributions from the object under study, the instrument, and the turbulent aberrations, i.e., 


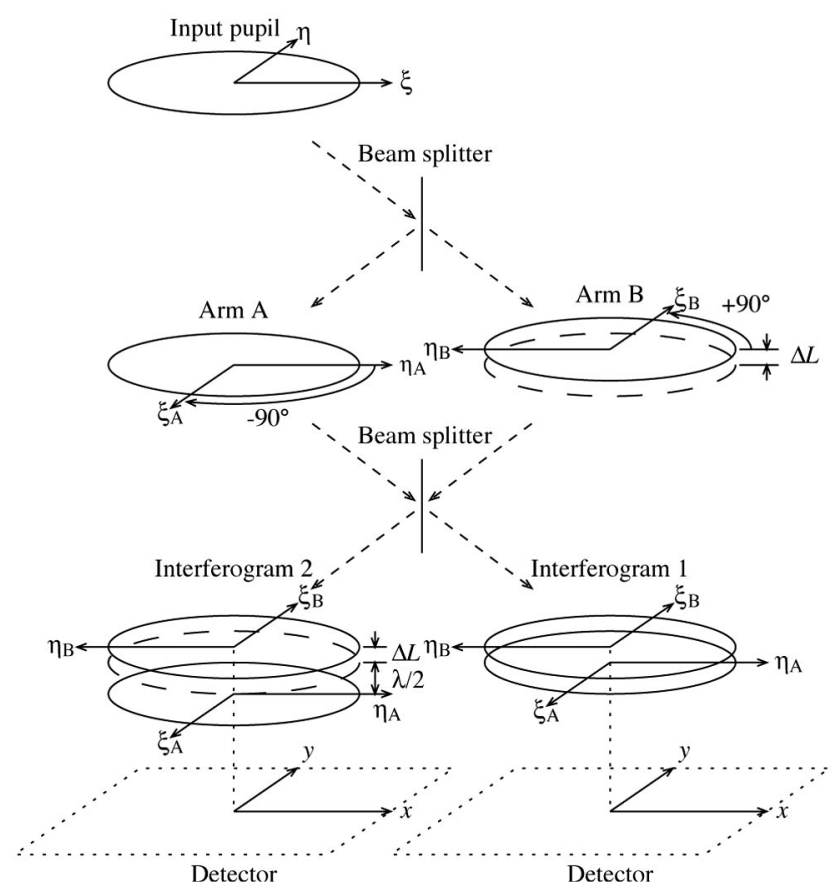

Fig. 2. Schematic rotation shearing geometry and coordinate systems. Light is incident on the input pupil at the top of the figure. Arms A and B each receive a copy of the input pupil, and rotate the copies 90 deg in opposite directions. When the two copies of the input pupil are recombined (through a beam splitter) they form two interferograms. Instrumental path-length differences, $\Delta L$, shown here as a constant with respect to $(\xi, \eta)$, effectively retard or advance the wavefronts in one arm. Vertical displacements in the figure represent path-length differences. The two output interferograms differ by $\pi \operatorname{rad}\left(180^{\circ}\right)$ in phase, by virtue of conservation of energy, shown here as a $\lambda / 2$ path-length difference in Interferogram 2. While two detectors are shown in this figure, the QPI is currently configured so that the two interferograms land side by side on the same detector.

$$
\phi_{\text {meas }}=\phi_{\text {obj }}+\phi_{\text {inst }}+\phi_{\text {turb }}
$$

Each of the terms in Eq. (7) can be expressed as a function of either $(x, y)$ interferogram coordinates or $(u, v)$ angular frequencies.

Some conventions are useful when describing the coordinate systems associated with QPI. Any quantity expressed in terms of interferogram coordinates, $(x, y)$, is due to the interference of light from two points in the input pupil. For example, the turbulent phase term, $\phi_{\text {turb }}(x, y)$, is the contribution of turbulent phase aberrations to the phase difference between interfering points in the input pupil. This contrasts with the typical definition of phase aberrations, $\varphi_{\text {turb }}(\xi, \eta)$, that are measured relative to a planar reference (such as the input pupil plane). While every interfering pair of $(\xi, \eta)$ points in the input pupil defines a pair of $(x, y)$ points in each interferogram, and the locations of $(\xi, \eta)$ points and the resulting $(x, y)$ interference are related by simple rotations, the individual contributions of the two $(\xi, \eta)$ points to any measured parameter cannot be disentangled by measurements in the interferogram. As such, the convention adopted here is that any parameter ex-

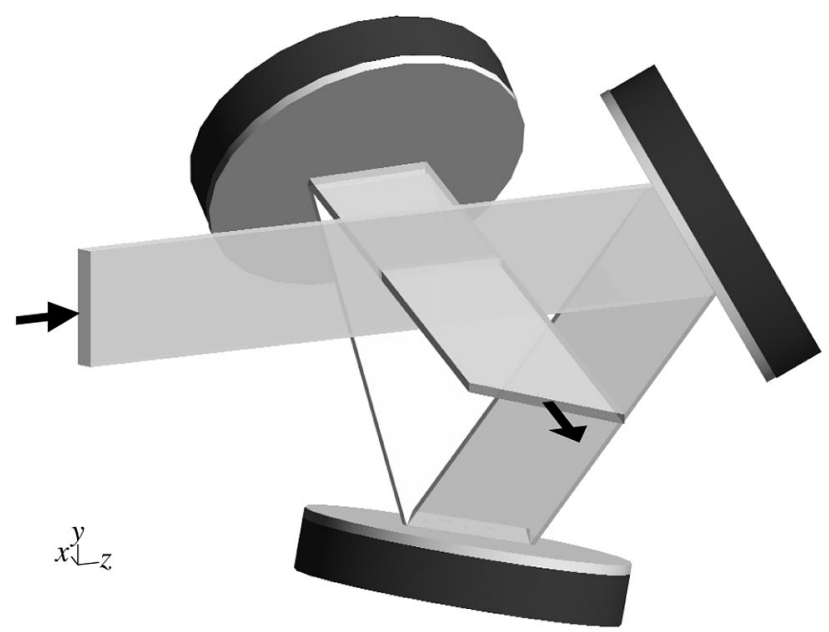

Fig. 3. Mirror arrangement giving rotation shear. Each arm of the interferometer contains three mirrors, arranged to fold the light path by $90 \mathrm{deg}$, and rotate the field of view by $\pm 90 \mathrm{deg}$ about the propagation direction. This figure depicts a fan of light rays, initially oriented vertically as it exits the first beam splitter (propagating in the $z$ direction in the displayed coordinate frame), being folded to propagate in the $-x$ direction and rotated to a horizontal orientation before entering the second beam splitter. The other arm of the interferometer performs the same rotation, in the opposite sense $(-90$ deg instead of $+90 \mathrm{deg}$ ). The angles of incidence of the chief ray on each of the three mirrors are the same.

pressed in $(x, y)$ coordinates represents a product of interference, which is not uniquely defined in $(\xi, \eta)$ coordinates. There is no conceptual difference between expressions in $(x, y)$ interferogram coordinates and $(u, v)$ angular frequencies (which are rotated and scaled by $\lambda)$, but, by convention, $(u, v)$ coordinates are only used after removal of instrumental terms, as when performing the Fourier transform to estimate $B(\alpha, \beta)$ from $\gamma(u, v)$.

The instrumental phase term in Eq. (7), $\phi_{\text {inst }}$, is determined by the optical path-length differences between the two arms of the interferometer and by the beamsplitter reflections and transmissions in the two

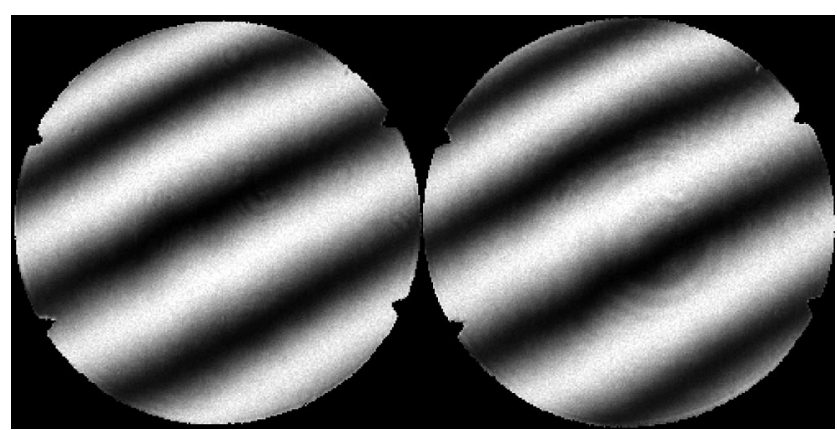

Fig. 4. Sample laboratory interferograms of a pinhole. Each point in the interferogram shows the interference of two points in the input pupil (see Fig. 1). The pinhole has a diameter smaller than the diffraction limit of the interferometer, giving a near-uniform visibility modulus. Corresponding points in the two interferograms differ in interferometric phase by $\pi$ rad. The constant phase gradient (manifested as fringes) shows that the object is off axis. No turbulent aberrations are present. 
arms. The optical path-length difference, $\Delta L(x, y)$, is a function of position in the interferogram (although it is shown as a constant in Fig. 2). For a given $(x, y)$ point, the effect of $\Delta L(x, y)$ is the same in both interferograms.

In addition to $\Delta L$, the instrumental phase contains a term representing the sequence of reflections and transmissions as light travels through the beam splitters, which is shown schematically as a $\lambda / 2$ pathlength difference in Interferogram 2 in Fig. 2. This difference in the instrumental phase provides the only difference between the measured quantities in Interferograms 1 and 2 . The instrumental phase difference between corresponding locations in the two full-pupil interferograms is exactly $\pi \mathrm{rad}\left(180^{\circ}\right)$. This phase difference can be interpreted as a consequence of conservation of energy (assuming no losses in the beam splitter) in that the intensity sum of the two full-pupil interferograms must represent all of the light entering the interferometer. At an $(x, y)$ point where one interferogram is bright, the same $(x, y)$ point on the other interferogram must be dark. It is misleading to represent this phase term as a $\lambda / 2$ path-length difference in Fig. 2, as this phase shift is achromatic, unlike the instrumental path-length difference $\Delta L$ shown in Fig. 2. The $\pi$ rad phase shift, which is a consequence of the conservation of energy, has no intrinsic relationship to the 180 deg rotation shear, which is a geometric design parameter. The $\pi$ rad phase shift is exact and would exist for any choice of rotation shearing angle.

The instrumental phase terms, incorporating both the path-length difference and the number of reflections, are

$$
\begin{aligned}
& \phi_{\text {inst }, 1}(x, y)=2 \pi \Delta L(x, y) / \lambda, \\
& \phi_{\text {inst }, 2}(x, y)=2 \pi \Delta L(x, y) / \lambda+\pi,
\end{aligned}
$$

where $\Delta L$ is the instrumental path-length difference, which is the same for both Interferograms 1 and 2, and the $\pi$ represents the phase shift due to an inequal number of reflections and transmissions.

\section{Quadrature Phase Interferometry}

The instrumental phase, $\phi_{\text {inst }}$, has no intrinsic symmetry properties with respect to the $(x, y)$ location. This differs from the object visibility phase and the turbulent phase (measured in the interferograms), both of which are antisymmetric about the centers of the interferograms. The antisymmetry of $\phi_{\mathrm{obj}}$ and $\phi_{\text {turb }}$ arises because they are phase differences of points in the input pupil, which appear with an opposite sign for diametrically opposed points in the interferograms.

The simplest quadrature-phase arrangement uses an instrumental path-length difference, $\Delta L(x, y)$, that is equal to $\lambda / 8$ everywhere. In this arrangement, for each point $(x, y)$ and its diametric opposite, $(-x,-y)$, we define

$$
\phi_{0}(x, y) \equiv \phi_{\mathrm{obj}}(x, y)+\phi_{\mathrm{turb}}(x, y)+\pi / 4 .
$$

This implies,

$$
\begin{aligned}
\phi_{\text {meas }, 1}(x, y) & =\phi_{\text {obj }}(x, y)+\phi_{\text {turb }}(x, y)+\pi / 4 \\
& =\phi_{0}(x, y), \\
\phi_{\text {meas }, 1}(-x,-y) & =-\phi_{\text {obj }}(x, y)-\phi_{\text {turb }}(x, y)+\pi / 4 \\
& =-\left[\phi_{0}(x, y)-\pi / 2\right], \\
\phi_{\text {meas }, 2}(x, y) & =\phi_{\text {obj }}(x, y)+\phi_{\text {turb }}(x, y)+5 \pi / 4 \\
& =\phi_{0}(x, y)+\pi, \\
\phi_{\text {meas }, 2}(-x,-y) & =-\phi_{\text {obj }}(x, y)-\phi_{\text {turb }}(x, y)+5 \pi / 4 \\
& =-\left[\phi_{0}(x, y)-3 \pi / 2\right],
\end{aligned}
$$

where $\phi_{\text {meas }, 1}(x, y)$ is the measured phase at $(x, y)$ in Interferogram 1 , and $\phi_{\text {meas }, 2}(x, y)$ is the measured phase at the same $(x, y)$ point in Interferogram 2 . The measured quantities in the interferograms are fluxes, which are given by Eq. (3) expressed in $(x, y)$ coordinates,

$$
\begin{array}{r}
I_{1}(x, y)=I_{0}(x, y)\left\{1+V_{\text {meas }}(x, y) \cos \left[\phi_{0}(x, y)\right]\right\}, \\
I_{1}(-x,-y)=I_{0}(x, y)\left\{1+V_{\text {meas }}(x, y) \sin \left[\phi_{0}(x, y)\right]\right\}, \\
I_{2}(x, y)=I_{0}(x, y)\left\{1-V_{\text {meas }}(x, y) \cos \left[\phi_{0}(x, y)\right]\right\}, \\
I_{2}(-x,-y)=I_{0}(x, y)\left\{1-V_{\text {meas }}(x, y) \sin \left[\phi_{0}(x, y)\right]\right\},
\end{array}
$$

where $I_{1}$ is the measured in Interferogram 1 and $I_{2}$ in Interferogram 2 . These measured quantities are used to estimate $I_{0}, \phi_{0}$, and $V_{\text {meas }}$, using

$$
\begin{aligned}
I_{0}(x, y) & =\left[I_{1}(x, y)+I_{2}(x, y)\right] / 2 \\
& =\left[I_{1}(-x,-y)+I_{2}(-x,-y)\right] / 2, \\
V_{\text {meas }}(x, y) & =\frac{\left\{\left[I_{1}(x, y)-I_{2}(x, y)\right]^{2}+\left[I_{1}(-x,-y)+I_{2}(-x,-y)\right]^{2}\right\}^{1 / 2}}{2 I_{0}(x, y)}, \\
\phi_{\text {obj }}(x, y)+\phi_{\text {turb }}(x, y) & =\tan ^{-1} \frac{I_{1}(-x,-y)-I_{2}(-x,-y)}{I_{1}(x, y)-I_{2}(x, y)}-\pi / 4 .
\end{aligned}
$$


By convention, $V_{\text {meas }}$ is nonnegative and the signs of the numerator and denominator of $\tan \phi_{0}$ unambiguously determine $\phi_{0}$ (modulo $2 \pi$ ). Equations (10) (12) describe the case when $\Delta L(x, y)=\lambda / 8$ for all $(x, y)$, which will not be generally true. In general, Eq. (12) takes on a more complicated form to account for the actual values of $\Delta L(x, y)$.

The quadrature-phase measurement is enabled by the point-antisymmetry of $\phi_{\text {obj }}$ and $\phi_{\text {turb }}$, and by the fact that the cosine function in Eq. (3), which determines the observed intensity, is even. For a given $(x, y)$ point, a quadrature-phase measurement utilizes the corresponding $(x, y)$ points from both interferograms, and the opposing $(-x,-y)$ points from both interferograms. Because $\phi_{\text {obj }}$ and $\phi_{\text {turb }}$ are antisymmetric, all of the available information is obtained by evaluating $V_{\text {meas }}$ and $\phi_{\mathrm{obj}}+\phi_{\text {turb }}$ over just half of the $(x, y)$ plane. It is then convenient to divide artificially each full-pupil interferogram into two halves at the $y=0$ line. In this sense, it can be considered that the interferometer produces four interferograms, which provide four measurements to estimate $V_{\text {meas }}$ and $\phi_{\text {obj }}+\phi_{\text {turb }}$. A sample set of interferograms is shown in Fig. 5, artificially manipulated (i.e., rotated by $\left.180^{\circ}\right)$ to align the $(-x,-y)$ axes for $y<0$ to the $(x, y)$ axes for $y>0$. In $(u, v)$ coordinates, the visibility is estimated for all $u>0$, which contains all of the available imaging information.

\section{E. Optical Design}

Because the interferograms and the image are related by a Fourier transform, the image resolution is determined by the overall size of the interferograms (i.e., by the pupil diameter, $D$ ), and the image field of view is determined by the interferogram resolution. Implicit in this statement is the fact that the $180 \mathrm{deg}$ rotation shear creates baselines whose lengths are related to interferogram location according to Eq. (6). The resulting image resolution is $\lambda / D$.

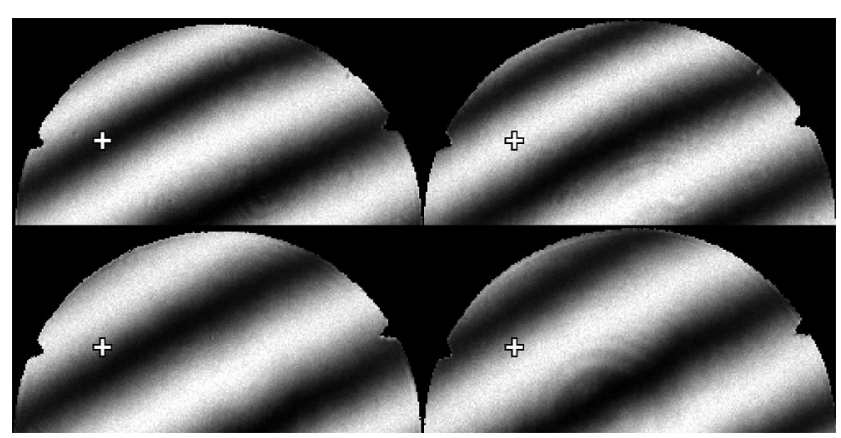

Fig. 5. Sample laboratory interferograms of a pinhole, divided into four interferograms. The upper panels are identical to the upper halves of the interferograms in Fig. 4, while the bottom panels have been rotated $180 \mathrm{deg}$ to emphasize the correlation between diametrically opposed points $(x, y)$ in the upper panels and $(-x,-y)$ in the lower panels. Four points that compose a quadrature-phase measurement are identified by crosses. The interferometric phases of the corresponding points in the left and right panels differ by $\pi \mathrm{rad}$, while those in the upper and lower panels differ by $\pi / 2 \mathrm{rad}$.
For objects far off center in the field of view, the spacing of the resulting fringes becomes small enough that the fringes are unresolved. To ensure Nyquist sampling of the fringes, the field of view must be limited to $(\lambda / D)\left(N_{\text {pix }} / 2\right)$, where $N_{\text {pix }}$ is the diameter of the interferogram measured in pixels (assuming the pixel size limits the interferogram resolution). As described in Subsection 3.C, $N_{\text {pix }}$ is typically either 512 or 256 , so the ratio of field of view to image resolution is either 256 or 128 . As an example, at $700 \mathrm{~nm}$ with a $5 \mathrm{~m}$ telescope, the diffraction limit is 0.03 arc sec, and the field of view is typically masked off at 3 arc sec-smaller than the more stringent Nyquist limit of interferogram resolution. The field of view is defined by a circular field stop so that light from outside the usable field of view does not appear in the interferograms and add noise. The limitation on image resolution and field of view does not depend on the turbulent conditions.

To facilitate object acquisition and tracking, a beam splitter is placed in the optical path before light enters the interferometer, to allow a direct image to be acquired simultaneously with the interferograms. This beam splitter reflects only $10 \%$ of the light to form the direct image. The detector (one of the CCDs described below) is square, and the two interferograms (which are circular) land side by side on the detector (see Fig. 4). This interferogram arrangement leaves much of the detector area unused, and so the direct image is also fed onto the same detector. This ensures that the direct image and the interferograms are synchronous, and that they are observed through identical turbulent aberrations.

\section{F. Imaging Extended Sources}

Interferometric imaging is performed in a fundamentally different manner from direct (focal-plane) imaging. The visibility modulus, which is directly measured interferometrically, can be interpreted as the deviation of the imaged scene from that of a point source. A point source gives a visibility modulus of unity everywhere, while a "flat field" (a uniformly illuminated field of view) gives a delta-function visibility modulus (unity at zero baseline, zero at all other baselines). Because the quadrature-phase interferometer is a homodyne interferometer operating at visible electromagnetic frequencies, Poisson noise from all light in the field of view is present in the measurement of the visibility modulus. This means that as the number of point sources in the field of view increases, the visibility modulus decreases and the measurement noise increases.

The signal to noise ratio for extended sources calculated from measurements of the real part of the complex visibility was analyzed by Ribak et al. ${ }^{19}$ That analysis included consideration of aliasing problems due to the incomplete visibility information, in that the resulting image formed from only the real part of the visibility is forced to be symmetric. The QPI measures the full complex visibility, and so is not subject to this same difficulty. Apart from that difference, the signal-to-noise analysis for the QPI follows that of 
Ribak et al. In practice, while extended images lead to lower signal-to-noise ratios, a QPI laboratory demonstration yielded high-quality images of a complicated, extended source (see Subsection 4.A).

\section{G. CCD Cameras}

This interferometer must capture short-exposure images $(<1 \mathrm{~s})$ to freeze the turbulent variations. In lowlight conditions, detector performance is determined by the read noise and the pixel readout rate. These two performance criteria are generally at odds with one another, requiring a compromise to be struck for a given observation. Two CCD cameras have been used with the QPI: a low-speed, relatively low-noise camera and a high-speed, relatively high-noise camera.

The high-speed camera was custom designed with multiple output amplifiers to minimize the peramplifier bandwidth and reduce the read noise as much as possible. It is a front-illuminated design, divided into 32 segments, each with its own output amplifier, as shown in Fig. 6. This enables frame rates up to 1000 frames per second, giving the highspeed CCD the name KFS-CCD, short for kiloframe per second CCD.

Each of the 32 segments consists of $512 \times 64$ optically active pixels, with each pixel measuring $12 \mu \mathrm{m} \times 12 \mu \mathrm{m}$, with a full well of $100000 \mathrm{e}^{-}$. Each segment contains an on-chip, three-stage, sourcefollower amplifier capable of supporting $40 \mathrm{Mpixel} / \mathrm{s}$ (1000 frames per second) data rates. The maximum power dissipated by the 32 on-chip amplifiers is $10 \mathrm{~W}$, which is typically cooled by $\mathrm{LN}_{2}$ to operate at $\sim 0{ }^{\circ} \mathrm{C}$.

The read noise of the KFS-CCD depends on the readout rate, temperature, and pixel binning. The shortest read time at which the performance has been optimized is $5 \mathrm{~ms}$ (200 fps), which gives $26 \mathrm{e}^{-} \mathrm{rms}$ noise unbinned and $19 \mathrm{e}^{-}$rms when binned $2 \times 2$. At $10 \mathrm{~ms}$ read times (100 fps), the noise performance is $20 \mathrm{e}^{-}$unbinned, $15.5 \mathrm{e}^{-}$binned $2 \times 2$. These numbers are higher by $2-4 \mathrm{e}^{-}$when uncooled.

The low-noise CCD is an off-the-shelf SITe SI502A back-illuminated, AR-coated $512 \times 512$ pixel CCD. The read noise is $5 \mathrm{e}^{-} \mathrm{rms}$ when cooled (nearly independent of readout rate), with a read time of $1.5 \mathrm{~s}$, which will be reduced to $0.5 \mathrm{~s}$ with the purchase of a new analog-to-digital converter (ADC) board. While the low-noise CCD readout is 2 orders of magnitude slower than the KFS-CCD and the number of pixels smaller by a factor of 4 , its quantum efficiency is higher by a factor of 2 and read noise lower by a factor of 3 , making its per-frame sensitivity significantly better. The KFS-CCD, however, is the preferred camera in cases where the addition of separate frames is efficient and the number of frames the KFS-CCD can acquire is sufficiently larger than the number the low-noise camera can acquire.

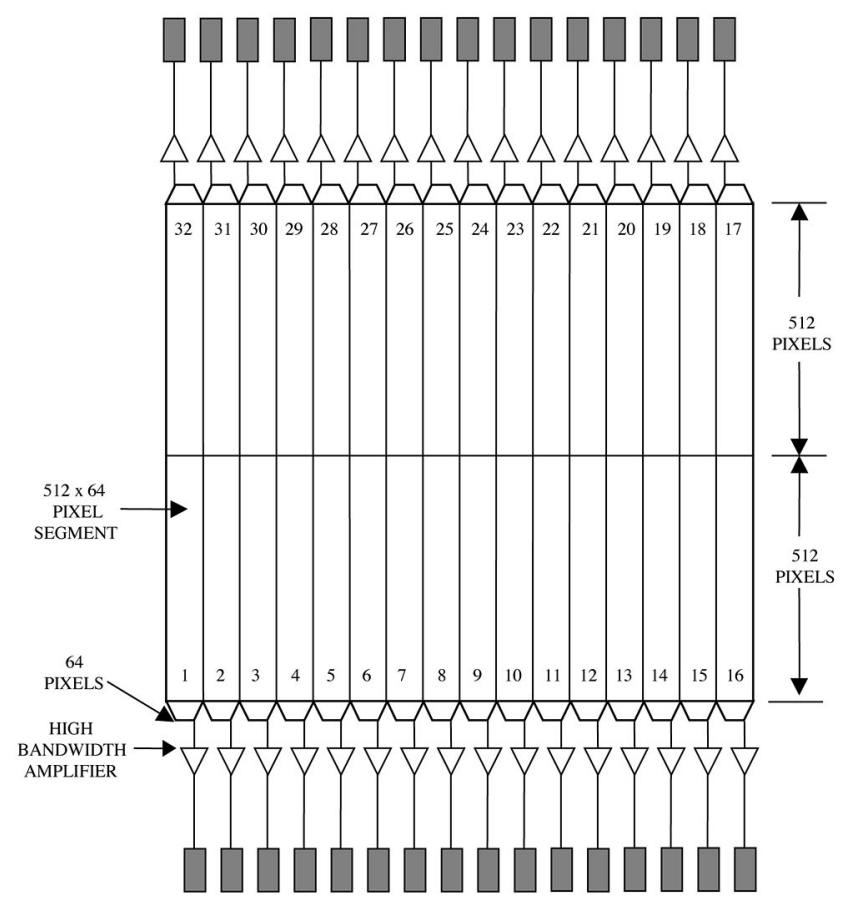

Fig. 6. Architecture of the KFS-CCD. The device is divided into 32 equivalent segments, each with a high-bandwidth floating diffusion amplifier.

\section{Effects of Turbulence}

\section{A. Phase and Amplitude Variations}

Index-of-refraction inhomogeneities due to turbulence add phase variations to the incident wave front. As the aberrated wave front propagates, the phase variations give rise to amplitude variations (scintillation). The turbulent phase variations are measured along with the object visibility phase as described by Eq. (12), while the amplitude fluctuations are measured as variations in $I_{0}(u, v)$. As can be seen in Eq. (12), the measured visibility modulus and phase are not affected by amplitude fluctuations, to first order.

\section{B. Visibility Modulus}

In the absence of noise, the measured visibility modulus is the product of several factors,

$$
V_{\text {meas }}=V_{\text {band }} V_{\text {temp }} V_{\text {spat }} V_{\text {amp }} V_{\text {obj }},
$$

where $V_{\text {band }}$ is the visibility loss due to finite bandwidth (coherence), $V_{\text {temp }}$ is temporal smearing (exposure time), $V_{\text {spat }}$ is spatial resolution (pixellation), $V_{\text {amp }}$ is the second-order visibility loss due to amplitude fluctuations (scintillation), and $V_{\text {obj }}$ is the object visibility modulus. All of the terms in Eq. (13) are potentially functions of $(u, v)$, and all are in the range $[0,1]$, which means the cumulative effect is always to reduce the measured visibility modulus (although measurement noise will bias $V_{\text {meas }}$ to higher values).

Equation (13) establishes several order-of-magnitude observing parameters. $V_{\text {band }}$ limits $\Delta \lambda / \lambda<2 \pi / \phi_{\text {rms }}$, 
where $\Delta \lambda$ is the spectral bandwidth and $\phi_{\mathrm{rms}}$ is the rms phase difference between points being interfered. In practice, for a typical $D / r_{0} \sim 50$ in Kolmogorov turbulence, this limits the fractional bandwidth to $\sim 1 / 10$ at the longest baselines. $V_{\text {temp }}$ limits exposure time to $\Delta x / U$, where $\Delta x$ is the pixel spacing (or the effective spatial resolution) and $U$ is the bulk wind speed. $V_{\text {spat }}$ will be discussed in Section $5 . V_{\text {amp }}$ is a second-order effect due to the fact that when scintillation is present, the wavefront amplitudes of a pair of points in the input pupil being interfered are not equal, while the visibility modulus calculation assumes they are equal. In conditions of strong scintillation, this limitation can be eliminated by separately recording pupil images (without interference), which can be done using a small fraction of the incident light, as the Fresnel zone sizes are likely to be large, allowing significant binning of the pupil image.

Under typical astronomical seeing conditions, the amplitude variations should be small, even at wavelengths as short as $400 \mathrm{~nm}$. However, in horizontal path propagation, or if a high-accuracy determination of $V(u, v)$ is required, the QPI can record pupil images using a parallel imaging channel (fed by a 90/10 beam splitter in front of the interferometer) that is normally configured as a direct-imaging channel for target acquisition and guiding. This mode of operation, recording noninterfered pupil images, allows direct calibration of $V_{\mathrm{amp}}$ in each exposure.

\section{Visibility Phase}

To form an accurate image from the measured data, the object visibility phase, $\phi_{\mathrm{obj}}$, must be distinguished from the turbulent-phase, $\phi_{\text {turb }}$. This problem is similar to those encountered in speckle imaging, which is normally solved using a Knox-Thompson algorithm ${ }^{20}$ or a phase-gradient algorithm. ${ }^{21}$ We use a phasegradient technique, which makes use of the correlation of turbulent-phase variations across short distances.

Turbulent-phase fluctuations are traditionally expressed as deviations from a planar wave front, measured in the input pupil. Phase fluctuations in the input pupil are expressed as $\varphi_{\text {turb }}(\xi, \eta)$, which determine $\phi_{\text {turb }}(x, y)$ when the corresponding $(\xi, \eta)$ points are interfered. A Kolmogorov spectrum of phase variations yields a structure function of phase fluctuations at the input pupil, ${ }^{3}$

$$
\begin{aligned}
D_{\varphi}(\rho) & =\left\langle\left[\varphi_{\text {turb }}(\xi, \eta)-\varphi_{\text {turb }}(\xi+\Delta \xi, \eta+\Delta \eta)\right]^{2}\right\rangle \\
& =6.88\left(\rho / r_{0}\right)^{5 / 3} \mathrm{rad}^{2},
\end{aligned}
$$

where $D_{\varphi}(\rho)$ is the mean square of the phase difference between two points in the input pupil separated by a distance $\rho=\left(\Delta \xi^{2}+\Delta \eta^{2}\right)^{1 / 2}$, averaged over all $(\xi, \eta)$ and all $(\Delta \xi, \Delta \eta)$. The turbulent phase measured for a given $(x, y)$ point, $\phi_{\text {turb }}(x, y)$, is the difference between the input phase fluctuations at the two points in the input pupil being interfered, so that

$$
\left\langle\phi_{\text {turb }}{ }^{2}(x, y)\right\rangle=D_{\varphi}\left[\left(x^{2}+y^{2}\right)^{1 / 2}\right],
$$

using Eqs. (4) and (14).

The phase sum estimated by Eq. (12) is only known modulo $2 \pi$, making it a "circular" quantity. The sample mean of a circular quantity whose variance is larger than approximately $1 \mathrm{rad}^{2}$ has extremely poor statistics, making it impractical to simply take the mean of $\phi_{\text {obj }}+\phi_{\text {turb }}$ to eliminate $\phi_{\text {turb }}$ (which has a mean value of zero). Instead, we form gradients by taking the difference in the phase in neighboring $(x, y)$ locations, so that $\left\langle\left[\nabla \phi_{\text {turb }}\right]^{2}\right\rangle$ is small and can be averaged out (here, $\langle\ldots\rangle$ denotes a temporal average over successive exposures).

Defining the $x$ gradient as $\nabla_{x} \phi(x, y)=[\phi(x$ $+\Delta x, y)-\phi(x, y)] / \Delta x$, the variance of the mean gradient is approximately

$$
\left\langle\left[\nabla_{x} \phi_{\text {turb }}(x, y)\right]^{2}\right\rangle \approx 2 D_{\varphi}(\Delta x) / \Delta x^{2},
$$

with an equivalent relationship for $\left\langle\left[\nabla_{y} \phi_{\text {turb }}(x, y)\right]^{2}\right\rangle$. Keeping $\Delta x^{2}\left\langle\left[\nabla_{x} \phi_{\text {turb }}\right]^{2}\right\rangle$ below $1 \mathrm{rad}^{2}$ sets a limit on the $(x, y)$ resolution,

$$
\Delta x<r_{0} / 5 \text {. }
$$

Using Palomar as an example, if $r_{0}=10 \mathrm{~cm}$, the maximum interferogram pixel spacing is $2 \mathrm{~cm}$, requiring 250 pixels to span the $5 \mathrm{~m}$ diam mirror. This allows both interferograms to be recorded, side by side, in a $512 \times 256$ pixel region, which in turn allows the KFS-CCD (with $1024 \times 1024$ pixels) to bin pixels $2 \times 2$ to reduce the total read noise and shorten the read time, while retaining sufficient spatial resolution.

As long as the condition on Eq. (17) is satisfied, the turbulent phase terms can be eliminated by averaging over a number of individual exposures, without first unwrapping the phase. This leaves a map of $\nabla_{x} \phi_{\mathrm{obj}}$ and $\nabla_{y} \phi_{\mathrm{obj}}$, assuming that the object visibility phase does not change with time. The visibility phase, $\phi_{\mathrm{obj}}$, can then be recovered from its gradients, e.g., by constructing $\nabla^{2} \phi_{\text {obj }}$ and solving Poisson's equation. The phase gradient technique can recover a phase map over contiguous regions of the $(x, y)$ plane, but cannot connect the disjoint regions. Depending on the size of the gaps between contiguous regions, it may be possible to relax the constraint set in Eq. (17) to connect disjoint regions of the $(x, y)$ plane. The practical limits of Eq. (17) have not been explored.

\section{Self-calibration}

Optimal interferometer alignment, giving $\Delta L(x, y)$ $=\lambda / 8$ as described in Eqs. (10)-(12), is not always possible. The surfaces of the mirrors that make up the interferometer, for instance, are not perfectly flat, so that the interferometer may be properly aligned at some $(x, y)$ locations but not at others. In addition, path-length differences can only equal $\lambda / 8$ for one value of $\lambda$, so if the interferometer is used at multiple 
wavelengths without realignment, it will only be properly aligned for one of those wavelengths.

If the instrumental phase between the two halves of each interferogram does not sum to $\pi / 2$, i.e., $\Delta L(x, y)+\Delta L(-x,-y) \neq \lambda / 4$, the equations used to estimate $V_{\text {meas }}$ and $\phi_{\mathrm{obj}}+\phi_{\text {turb }}$ incorporate the instrumental phase offset, $\phi_{\text {off }}(x, y)=(2 \pi / \lambda)[\Delta L(x, y)$ $+\Delta L(-x,-y)]$, defined for $y>0$. For a given level of noise, the estimation of $V$ and $\phi_{\text {obj }}+\phi_{\text {turb }}$ are most accurate when $\phi_{\text {off }}$ is near $\pm \pi / 2$, and least accurate when $\phi_{\text {off }}$ is near 0 or $\pi$. Separately from the determination of $\phi_{\text {off }}$, deviations of $\Delta L(x, y)$ from $\lambda / 8$ (for $y>0)$ act as an additive term to the estimate of $\phi_{\text {obj }}+\phi_{\text {turb }}$ using Eq. (12). It is useful to separate conceptually the determination of instrumental phases into the determination of $\phi_{\text {inst }, 1}(x, y)$ for $y$ $>0$, and the determination of $\phi_{\text {off }}(x, y)$.

Both of these terms can be determined using an internal calibration lamp, that collimates light from a pinhole and sends it through the instrument. Alternately, if $\phi_{\text {off }}(x, y)$ is known a priori, external calibration using observations of a point source (or any object with known structure) can determine $\phi_{\text {inst }, 1}(x, y)$.

In the presence of turbulent-phase fluctuations with amplitudes much larger than $2 \pi$, $\phi_{\text {off }}(x, y)$ can be determined, up to a \pm sign, from any data set. This self-calibration makes use of the identity $1 /(2 \pi) \int_{0}^{2 \pi} \cos \theta \cos (\theta+\Delta \theta) \mathrm{d} \theta=\cos (\Delta \theta) / 2$ to say that was imaged under weakly aberrating conditions $\left(D / r_{0} \sim\right.$ few $)$. In the astronomical tests, simple objects were imaged under moderately aberrating conditions $\left(D / r_{0} \sim 40\right)$. Observations of Vega (an unresolved point source) and of Capella (a binary star) provided useful data during the astronomical tests.

\section{A. Laboratory Test}

In the laboratory tests, a short-arc mercury lamp illuminates the test object, the light from which is collimated, passed through a pupil stop, and fed into the interferometer, as shown in Fig. 7. A turbulent He-air jet is then passed through the collimated beam, introducing turbulent phase and amplitude variations. A chopper wheel limits exposure times to $200 \mu \mathrm{s}$, short enough to freeze the turbulent aberrations in each exposure. An optical filter limits the spectral bandpass to a single $\mathrm{Hg}$ spectral line at $577 \mathrm{~nm}$. Direct images and interferograms are recorded simultaneously to compare the imaging performance of the interferometer to that of direct imaging.

Without the turbulent $\mathrm{He}$-air jet, the imaging performance of the direct and interferometric images is comparable, as seen in the top panels of Fig. 8. The pixel scale in the direct image does not Nyquistsample the diffraction limit, resulting in some loss of direct image resolution. When the turbulent He-air jet is introduced, the interferometric image quality is

$$
\cos \phi_{\text {off }}(x, y)=4 \frac{\left\langle\frac{\left[I_{1}(x, y)-I_{2}(x, y)\right]\left[I_{1}(-x,-y)-I_{2}(-x,-y)\right]}{\left[I_{1}(x, y)+I_{2}(x, y)\right]\left[I_{1}(-x,-y)+I_{2}(-x,-y)\right]}\right\rangle}{\left\langle\frac{\left[I_{1}(x, y)-I_{2}(x, y)\right]^{2}}{\left[I_{1}(x, y)+I_{2}(x, y)\right]^{2}}\right\rangle+\left\langle\frac{\left[I_{1}(-x,-y)-I_{2}(-x,-y)\right]^{2}}{\left[I_{1}(-x,-y)+I_{2}(-x,-y)\right]^{2}}\right\rangle} .
$$

This equation, while seemingly complicated, involves only the measured intensities of sets of corresponding points in the four virtual interferograms, and can be obtained from observations of any object. Moreover, this calibration is independently determined for every $(x, y)$ point (for $y>0$ ).

Using this self-calibration technique, the quadrature-phase measurements can always be estimated using instrumental phase terms determined from the observational data itself. In other words, no separate calibration data need to be taken to accurately determine $\phi_{\text {off }}$ because it can be determined from any data during analysis, provided that $\phi_{\text {turb }}$ has sufficiently large variance and that $\phi_{\text {obj }}$ does not change on similar timescales.

\section{Performance}

The interferometer has been tested in the laboratory under controlled conditions, and at Palomar Observatory under ordinary nighttime observing conditions. In the laboratory tests, a complicated object only slightly degraded, while the aberrations dramatically blur the direct images.

In the configuration used, the diameter, $D$, of the pupil stop where the He-air jet was Introduced is $8 \mathrm{~mm}$. This small $D$ yields $D / r_{0}$ on the order of $5-10$, which does not constitute as demanding an aberrating environment as the interferometer is designed for $\left(D / r_{0} \sim 50-100\right)$. Rather than using the full phase gradient technique described in Subsection 3.C, the interferometric phase correction employed to correct these low-order aberrations is to subtract the spatially averaged phase gradient from the measured phases. This is equivalent to performing a tip-tilt correction.

The quality of the laboratory interferometric images, given that only a first-order correction technique was employed, demonstrates an intrinsic strength of the rotation shearing interferometer. The $180 \mathrm{deg}$ rotation shear ensures that point-symmetric aberrations are eliminated from the measurements. This prevents defocus, spherical aberration, astigma- 


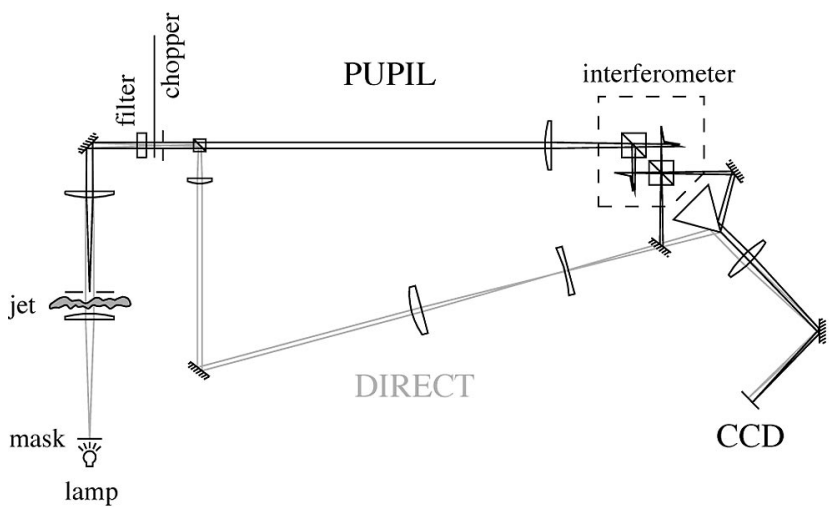

Fig. 7. Optical layout for the laboratory test. A Hg lamp illuminates a mask, and the light is collimated and stopped down. Turbulent aberrations are introduced into the collimated light by a He-air jet. A beam splitter breaks off $10 \%$ of the light as a direct image, shown here shaded as the DIRECT component, while the rest of the light passes through the interferometer, shown here as the PUPIL component. The CCD records the direct image and the two interferograms simultaneously (near the CCD, the direct image and one interferogram overlap as drawn, but are separated vertically). The mirrors in the interferometer, shown in Fig. 3, are not drawn here.

tism, etc. from affecting the interferometric images. In an environment where $D / r_{0}$ is large, this insensitivity has relatively little effect, but when $D / r_{0}$ is small, this point-symmetric insensitivity, combined with a tip-tilt correction, allows good imaging performance. The aberrated image quality can be further improved over that in Fig. 8 by making full use of the phase gradient technique described in Subsection 3.C rather than only the first-order correction shown.

The complexity of the reconstructed image shows that while the signal-to-noise ratio of an extended scene is reduced because of the low visibility modulus and large amount of Poisson noise (see Subsection 2.F), complicated objects can still be imaged with this technique. The equivalent number of diffractionlimited point sources that combine to make the image shown in Fig. 8 is approximately 1000. This experiment is likely to be made more difficult when applied to real imaging environments of interest, where $D / r_{0}$ may be quite large, but the signal-to-noise reduction due to the extended nature of the source does not preclude the use of this technique on such objects.

\section{B. Astronomical Observations}

The quadrature-phase interferometer operated at the coudé focus of the Palomar $5 \mathrm{~m}$ telescope in July 2002 under $\sim 1$ arc sec $R$-band seeing conditions. Telescope flexure was greater than anticipated, and the large motions of the input pupil complicated the interferometer alignment. Usable data were obtained on Vega, a bright unresolved, pointlike source, and Capella, a narrow-separation binary star system. These observations were made at a center wavelength of $700 \mathrm{~nm}$ with a $40 \mathrm{~nm}$ bandpass (this fractional bandwidth of 0.06 is conservative relative to the order-of-magnitude bandwidth limit of $\sim 1 / 10$ as
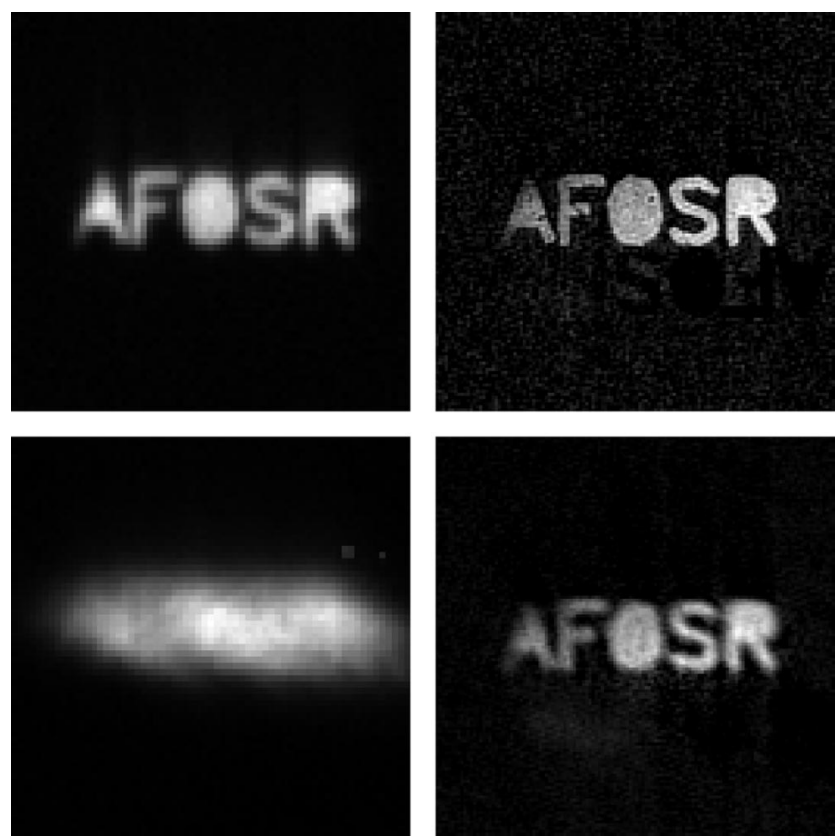

Fig. 8. Direct and interferometric laboratory images. The left panels are direct images, and the right panels are interferometric images. Corresponding direct and interferometric images are acquired simultaneously through identical conditions. The top panels include no turbulent aberrations, and the bottom panels are imaged through a turbulent $\mathrm{He}$-air jet that introduces phase and amplitude aberrations. Each of the bottom panels is an average of the same ten exposures. The interferometric image quality is only slightly affected by the aberrations, while the aberrated direct images are blurred beyond recognition. The diffraction limit of the system is approximately half the width of the line segments making up the letters.

described in Subsection 3.B), in $12.5 \mathrm{~ms}$ exposures followed by $12.5 \mathrm{~ms}$ readouts.

In the process of realigning the interferometer, the rotation shear deviated from $180 \mathrm{deg}$. As such, a quadrature phase relationship did not exist in the data taken on Capella. Without a quadrature phase relationship, visibility modulus measurements were made but no visibility phase measurements could be made, and so true images could not be formed of Capella. This misalignment was corrected for the observations of Vega.

At the time of the observations, the KFS-CCD data acquisition system filled internal memory buffers, then stopped acquisition and transferred the stored data to disk. The buffers were capable of storing 400 exposures, which at a rate of $40 \mathrm{~Hz}(12.5 \mathrm{~ms}$ exposures and $12.5 \mathrm{~ms}$ read times) gave $10 \mathrm{~s}$ contiguous datasets (at a 50\% duty cycle). In the time since these observations were made, this limitation has been lifted.

\section{Vega Observations}

A simple analysis has been performed on a single $10 \mathrm{~s}$ data set of Vega interferograms. A sample interferogram is shown in Fig. 9. Phase gradients are averaged as described in Subsection 3.C and $\phi_{\mathrm{obj}}$ is estimated from the mean gradients. The complex ob- 


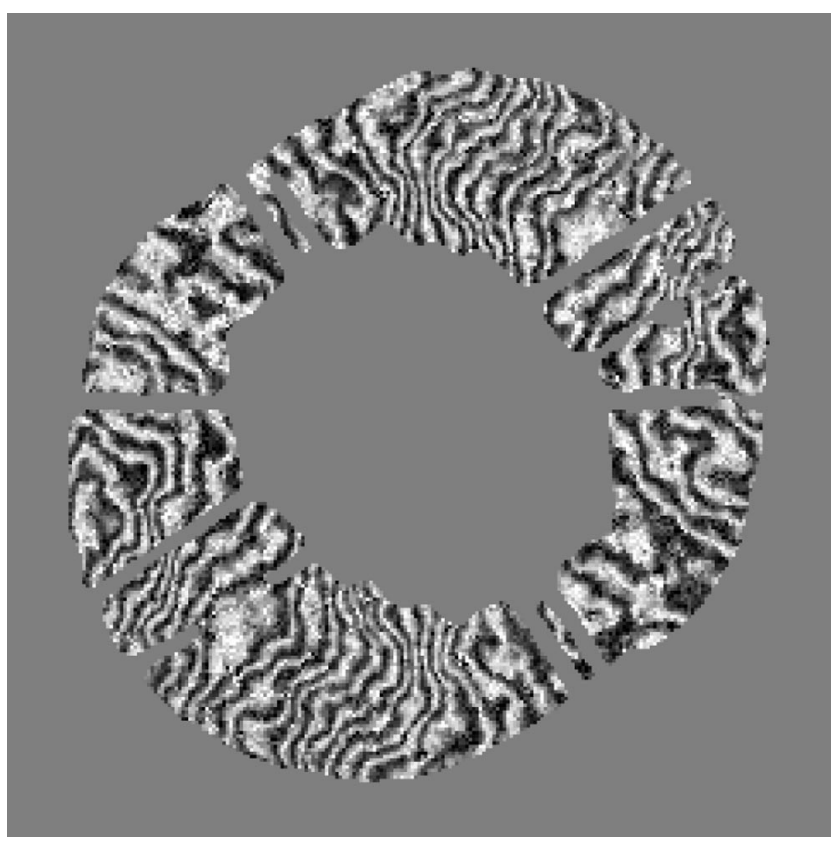

Fig. 9. Sample differenced interferogram of Vega. This figure is the difference between Interferogram 1 and 2 for a single exposure, removing scintillation effects. The visibility modulus is essentially uniform across the exposure. The input pupil was not correctly aligned with the interferometer center of rotation, leading to an oversized central obscuration and noncircular outer boundary.

ject visibility is then Fourier transformed to give an image. This raw image is shown in the middle panel of Fig. 10. This image has a full width at half maximum (FWHM) of 0.5 arc sec, smaller than the direct image FWHM of 1.0 arc sec (seeing limited), but far larger than the diffraction-limited resolution of 0.029 arc sec. The instrumental phase offset, $\phi_{\text {off }}$, was calculated from the data, ensuring proper quadrature-phase relationships, but no estimate was made of $\phi_{\text {inst, } 1}$ to remove instrumental phase terms from the quadrature measurements.

Using the same $10 \mathrm{~s}$ dataset, a simple estimate can be made of the image quality due to phase noise alone, by subtracting the estimated object phase of the second $5 \mathrm{~s}$ of the data set from the object phase of the first $5 \mathrm{~s}$, removing any instrumental phase contributions. The image formed from this difference is shown in the right-hand panel of Fig. 10, which displays a FWHM of 0.2 arc sec. This is analogous to using $5 \mathrm{~s}$ of data as an external calibration, while observing a point source, to correct the instrumental phase terms in another $5 \mathrm{~s}$ data set. This is not a true image of Vega, of course, because the object phase should be constant over the entire $10 \mathrm{~s}$, and therefore this represents the image of an object with $\phi_{\text {obj }}(u, v)=0$. To the extent that Vega is unresolved, this is a valid exercise.

An analysis of the temporal autocorrelation of the phase gradients shows long-lived correlations in $\phi_{\text {turb }}$. The temporal autocorrelation shows a roughly exponential decay with time lag, showing a characteristic
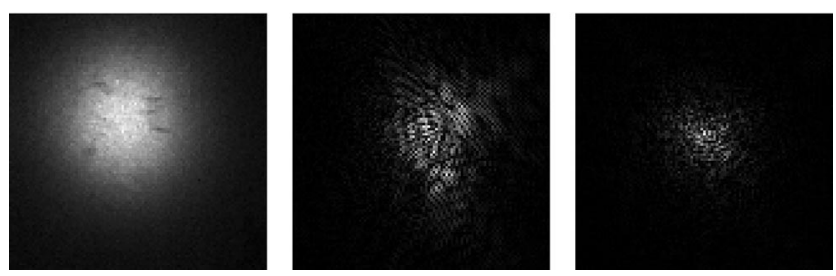

Fig. 10. Direct, raw interferometric, and differenced interferometric images of Vega. The left panel is the average of $10 \mathrm{~s} \mathrm{(400}$ frames) of direct images of Vega. The middle panel is a raw interferometric image, from the same $10 \mathrm{~s}$ of data as the left panel, formed without estimating the instrumental phase. The right panel is formed by calibrating one half of the $10 \mathrm{~s}$ dataset using the other half. The FWHM of these images is 1.0, 0.5, and 0.2 arc sec.

$(1 / e)$ time scale of $0.65 \mathrm{~s}$. This characteristic time scale, when compared to a characteristic length scale of $r_{0} \sim 14 \mathrm{~cm}$, implies a characteristic speed of $0.2 \mathrm{~m} / \mathrm{s}$. This low speed is indicative of dome seeing or other turbulent motions not related to the bulk wind velocity outside of the telescope dome (likely to be larger by an order of magnitude).

The variance of the mean phase gradient depends on the number of phase-gradient samples and the correlation between those samples. With long-timescale correlations between phase-gradient samples, the effective number of independent samples is quite small, and the resulting mean phase gradient retains a large variance, as seen by the FWHM of the reconstructed images. As an order of magnitude approximation, $10 \mathrm{~s}$ of phase gradient data with a $0.65 \mathrm{~s}$ correlation time scale yields $\sim 15$ independent samples, reducing the rms phase noise by a factor of $\sim 4$, comparable to the decrease in FWHM from 1.0 to 0.2 arc sec.

The interpretation of these results is that the turbulent-phase aberrations present were dominated by dome-level atmospheric structures (i.e., slowmoving structures), which necessitates a longer observing time span to separate $\phi_{\mathrm{obj}}$ from $\phi_{\text {turb }}$. Accordingly, longer individual exposure times could have been used, increasing the sensitivity with little loss of visibility.

\section{Capella Observations}

Capella is a binary system, with a 100-day orbital period and semimajor axis 0.05 arc sec. This separation is unresolved under ordinary 1 arc sec seeing conditions, but well beyond the 0.03 arc sec diffraction limit of the $5 \mathrm{~m}$ telescope at $\lambda=700 \mathrm{~nm}$.

As previously mentioned, we did not obtain quadrature-phase measurements of Capella. The visibility modulus is estimated from $10 \mathrm{~s}$ of data. The visibility modulus of a binary star system is sinusoidal, and the measured visibility modulus is fit to a sinusoid to determine the vector separation of the binary pair. Because only the visibility modulus was used, the orientation is uncertain by $180 \mathrm{deg}$. The measured separation is shown in Fig. 11, overlaid on the orbit as measured by long-baseline interferometry. ${ }^{22}$ The difference between the measured separation and the 


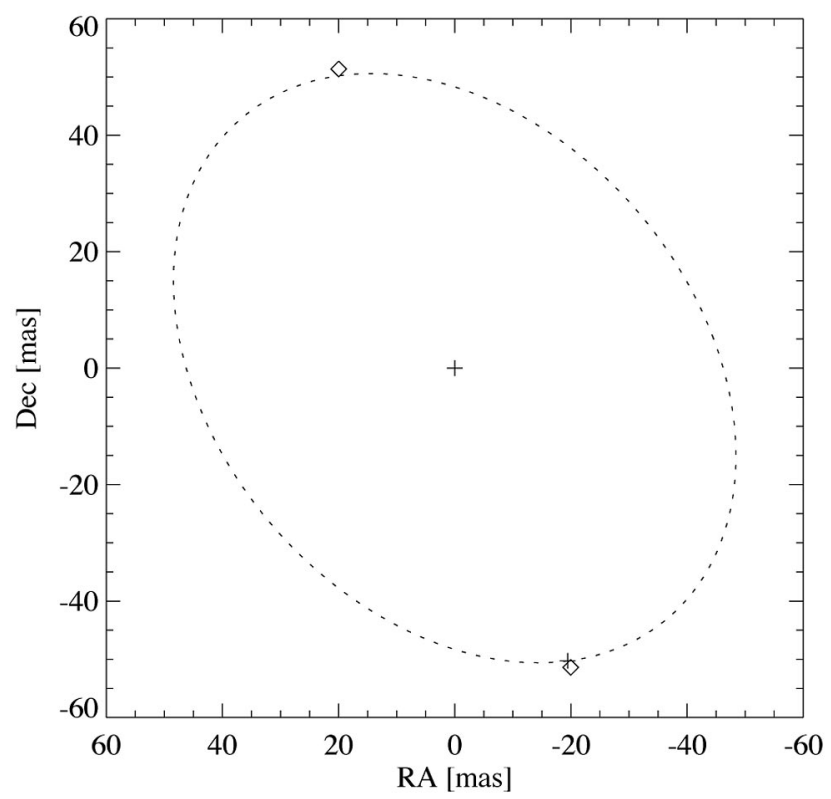

Fig. 11. Binary separation of Capella. The diamonds mark the separation measured by the QPI, with two diamonds shown because the separation is uncertain by $180 \mathrm{deg}$. The crosses mark the origin and the expected binary separation at the time of the observation, with the dotted curve tracing out an entire orbit. The separation is measured by fitting the measured visibility modulus from $10 \mathrm{~s}$ of data to a sinusoid, appropriate to a binary pair. The disagreement between the measured and expected binary separations is 0.0012 arc sec.

expected separation at the time of the observation was 0.0012 arc sec (excluding the possibility that the measurements disagree by $180 \mathrm{deg}$ ). This disagreement is considerably smaller than the semimajor axis of the binary orbit, 0.05 arc sec, and than the diffraction limit of the telescope, 0.03 arc sec.

\section{Expected Sensitivities}

A simple estimate of the sensitivity of QPI can be made by requiring an intensity signal-to-noise ratio of unity in each pixel in each frame. For Palomar, assuming $D$ $=5 \mathrm{~m}, \lambda=700 \mathrm{~nm}, \Delta \lambda=40 \mathrm{~nm}$ (this bandwidth is more conservative than the estimate in Subsection $3 . \mathrm{B}), r_{0}=15 \mathrm{~cm}$, and $v=10 \mathrm{~m} / \mathrm{s}$, the interferogram pixel scale could be $\Delta x=3 \mathrm{~cm}$, with an exposure time $t_{\text {exp }}=15 \mathrm{~ms}$. Using the KFS-CCD (the high-speed camera), with a detection efficiency of $15 \%$ and a CCD read noise of $15.5 \mathrm{e}^{-} /$pix, the point-source sensitivity limit is $R=5.5$. At Keck with $D=10 \mathrm{~m}$ and $r_{0}$ $=30 \mathrm{~cm}$, the sensitivity limit is $R=7.8$. For terrestrial (ground-to-ground) imaging with $D=0.4 \mathrm{~m}$, $r_{0}=8 \mathrm{~mm}$, and $v=10 \mathrm{~m} / \mathrm{s}$, the point-source sensitivity limit is $\sim 10^{4}$ phot $\mathrm{cm}^{-2} \mathrm{~s}^{-1} \AA^{-1}$, which is well below the flux of reflected sunlight $\left(\sim 10^{6}\right.$ phot $\mathrm{cm}^{-2} \mathrm{~s}^{-1} \AA^{-1}$ from a source the size of the telescope diffraction limit), but above the flux of scattered sunlight $\left(\sim 10^{2}\right.$ phot $\mathrm{cm}^{-2} \mathrm{~s}^{-1} \AA^{-1}$ from a source the size of the telescope diffraction limit).

Using the unity signal-to-noise ratio rule of thumb, near the faint end of the sensitivity limits the low- noise CCD provides superior performance to that of the KFS-CCD, in the sense that individual exposures retain adequate signal-to-noise ratios. With a detection efficiency of $30 \%$ and read noise of $5 \mathrm{e}^{-} / \mathrm{pix}$, the Palomar sensitivity limit is $R=7.8$ (under the same conditions described previously), and the Keck sensitivity limit is $R=10$. For terrestrial imaging, the sensitivity limit is $\sim 10^{3}$ phot $\mathrm{cm}^{-2} \mathrm{~s}^{-1} \AA^{-1}$, which would be comparable to the limit for scattered sunlight off an extended scene, whose illuminated area covers $\sim 10$ diffraction-limited resolution elements (a much simpler scene than used in the demonstration in Fig. 8, which imaged $\sim 1000$ diffraction-limited resolution elements).

The high measured visibility modulus of the Vega observations implies that the exposure time, resolution, and bandwidth can be increased beyond their rule-of-thumb limits to increase sensitivity at the expense of some additional calibration requirements.

The sensitivities quoted above are highly simplified. The ultimate sensitivity of an observation depends on the ability to reduce the variance of the mean phase gradient (and mean visibility modulus), which may depend on the atmospheric conditions, as structures causing phase aberrations may persist over many exposures. Assuming that phase errors dominate image resolution, image resolution increases with observing time. For objects brighter than the limits above, the KFS-CCD is often preferred because it allows many more exposures in a given period of time, thereby reducing phase errors.

\section{Discussion}

Depending on the imaging application, the primary strengths of quadrature-phase interferometry may be its high wavefront resolution, passive operation, insensitivity to amplitude fluctuations, operation at short wavelengths, or ability to perform internal calibration. The interferometric $(u, v)$ coverage obtained by this technique is nearly complete, allowing for reconstruction of highly detailed, near diffractionlimited images.

The imaging performance of quadrature-phase interferometry can be compared to AO and speckle interferometry. Compared to AO, QPI is simple, inexpensive, and capable of working at visible wavelengths (with $D / r_{0}>50$ ), but is less sensitive and generally observes a narrower field of view. Assuming a similar wavelength of operation, $\mathrm{AO}$ is more sensitive because the $\mathrm{AO}$ wavefront sensors are not subject to the same resolution criterion as QPI for phase-gradient recovery [Eq. (17)]. By using fewer pixels to record the same wavefront area, they incur less read noise per measurement. Once the AO control loop is closed, the corrected image is sent to a science camera that can take long exposures where read noise is not a limiting factor. When imaging in the presence of strong scintillation, however, QPI should be insensitive to the wavefront amplitude fluctuations, while AO would suffer some image degra- 
dation. This potential advantage of QPI has not yet been verified.

Unlike AO, QPI does not make direct measurements of the turbulent-phase fluctuations at the input pupil, $\varphi_{\text {turb }}(\xi, \eta)$. The turbulent-phase terms that the QPI does measure, $\phi_{\text {turb }}(x, y)$, are differences of phase fluctuations at two points in the input pupil, $\varphi_{\text {turb }}(\xi, \eta)$. It is not possible with the arrangement described here to uniquely determine $\varphi_{\text {turb }}(\xi, \eta)$ using QPI. While this precludes direct wavefront measurements, the QPI does directly measure the turbulentphase structure function, $D_{\varphi}$, so while QPI can directly determine the statistical properties of the turbulent-phase fluctuations, it cannot determine any individual realizations of $\varphi(\xi, \eta)$. The measurement of $D_{\varphi}$ using QPI does not require any assumptions about Kolmogorov turbulence or homogeneity or isotropy, only of translational invariance in the $(\xi, \eta)$ plane. It is interesting to note that rotation shearing interferometry is inherently unaffected by point-symmetric aberrations, such as spherical aberration, defocus, astigmatism, etc.

Compared to speckle interferometry, QPI has comparable sensitivity and simpler calibration requirements, but is more expensive and optomechanically more complex. The practical sensitivity of QPI is likely to be lower than that of speckle, because the QPI optical design involves more reflections, resulting in a lower instrumental throughput. The calibration requirements of speckle interferometry are highly dependent on the turbulent conditions, as they essentially involve measuring the modulation transfer function (MTF) degradation due to the turbulent aberrations, and statistically correcting the measured MTF to its unaberrated level. The MTF of pupil-plane interferometry, on the other hand, is essentially unity for all frequencies up to the diffraction-limited frequency $(D / \lambda) \cdot{ }^{23}$ QPI suffers a loss of visibility due to finite exposure times, wavefront resolution, and spectral bandwidth, as described in Eq. (13), but as seen from the observations of Vega, the measured visibility modulus is still near unity, requiring little calibration. The QPI instrumental phase calibration can be done internally and though the self-calibration of imaging data, although the observations of Vega did not demonstrate this ability.

Previous rotation shearing interferometers were inefficient primarily in three ways. First, the only way to measure visibility phase instantaneously was to add a large phase gradient (wavefront tilt) to the interferograms and measure deviations from the gradient, which limits the spectral bandwidth and generates systematic uncertainties. If an interferometer does not measure phase instantaneously, the measurement is confused by phase changes between exposures. ${ }^{12}$ Second, all previous rotation shearing interferometers used in astronomical observations have used only half of the incident light, reflecting one interferogram back toward the telescope. As a third, minor point, some rotation shearing interferometers used internal reflections in roof prisms to generate rotation shear, which introduced a polarization mismatch between the two arms, reducing the measured visibility modulus unless a corrector plate was introduced in the system. The QPI avoids these limitations; the first two problems are avoided by design, while the third (polarization mismatch giving low-visibility moduli) is bounded by the raw measured visibility modulus in interferograms such as those in Fig. 4, which have $V>0.97$ in unpolarized light.

Alternate modes of operation are possible with QPI. As demonstrated with observations of Capella, QPI can be operated in a modulus-only mode. While the Capella observations did not make use of the quadrature-phase relationships of QPI, a planned modulus-only measurement would make quadraturephase measurements of modulus and phase, but would relax the phase-gradient resolution limit in Eq. (17) to $\Delta x \sim r_{0}$, which relaxes the magnitude limits listed above by approximately 3.5 magnitudes, so that the low-noise CCD could observe objects down to approximately $R=11.3$ at Palomar and $R=13.5$ at Keck. No attempt would be made to separate $\phi_{\text {obj }}$ from $\phi_{\text {turb }}$, but the visibility modulus would be accurately measured at all baselines in each exposure. These expected sensitivities can be compared to the demonstrated speckle interferometry magnitude limit of $V$ $<12.5$ for binary star measurements at the U.S. Naval Observatory $0.66 \mathrm{~m}$ refractor. ${ }^{24}$ While it may seem inappropriate to compare a sensitivity limit on a $0.66 \mathrm{~m}$ telescope to expected sensivities on 5 and $10 \mathrm{~m}$ telescopes, the expected sensivities have no dependence on $D$, only on $r_{0}$. Use of large telescopes, such as those at Palomar and Keck, greatly improves the diffraction limit for a given $\lambda$, but does not change the expected sensitivity limit for a given $r_{0}$.

A potential upgrade to QPI would allow differential phase measurements as a new mode of operation. Differential phase measurements are enabled by adding spectral dispersion and reducing the interferogram spatial resolution. The simplest way to achieve this is to add a dispersive grating and a lenslet array. The lenslets reduce the interferogram spatial resolution, and the grating spectrally disperses the light from each lenslet as it lands on the detector. Quadrature-phase relationships are independently measured for each wavelength, and phase differences between wavelengths can be accurately measured. When observing objects smaller than the diffraction limit, the differential phase measurements determine the relative displacements of the photocenter at different wavelengths. This could be used to measure physical scales in broad-line regions of active galactic nuclei or orbits of extrasolar planets with strong absorption lines.

\section{Conclusions}

The fundamental operation of a quadrature-phase interferometer has been demonstrated, to varying degrees, in laboratory and astronomical tests. The capabilities of this technique under the viewing conditions of interest, observing complicated objects un- 
der astronomical viewing conditions or objects under strongly aberrating conditions, have not yet been demonstrated.

This research was funded in part by the Air Force Office of Scientific Research (AFOSR) Grants No. F49620-94-1-0283, F49620-00-1-0036, F49620-03-10102, F49620-98-1-0052, and DoD-DURIP/AFOSR Grant No. F49620-95-1-0199. This material is based on work supported by the National Science Foundation under Grant No. AST9618880. This work was performed in part under contract with the Jet Propulsion Laboratory (JPL) funded by NASA through the Michelson Fellowship Program. JPL is managed for NASA by the California Institute of Technology. Based partly on observations obtained at the Hale Telescope, Palomar Observatory, as part of a collaborative agreement between the California Institute of Technology, its divisions Caltech Optical Observatories, and the Jet Propulsion Laboratory (operated for NASA), and Cornell University. Bryan Jacoby, Stephen Kaye, and Ryan McLean were invaluable in fielding the QPI.

\section{Appendix A: QPI Optical Implementation}

The optical design described here for creating quadrature-phase interferograms is not unique. The original implementation of the QPI had a more complicated optical arrangement.

In the original implementation of the QPI, one mirror in one arm of the interferometer was split into two halves. The division between mirror halves was positioned so that the split between the mirrors ran through the center of the interferograms. The instrumental phase terms were independently adjustable for each of the two halves. In this configuration, the division of the two full-pupil interferograms into four interferograms, shown graphically in Fig. 5, was straightforward, as the interferograms were already split in half.

The advantage of the split arrangement is that it allows any instrumental path-length difference $\Delta L$, to be used on each half of the interferograms. The simplest choice for $\Delta L(x, y)$ is 0 on one half, i.e., for $y>0$, and $\lambda / 4$ on the other (for $y<0$ ), enabling quadrature-phase measurements. An additional advantage of having independent alignment of the two halves is that the adjustment resolution of each mirror half determines the adjustment resolution of $\phi_{\text {inst }}(x, y)+\phi_{\text {inst }}(-x,-y)$, while in the current configuration, with just one set of adjustments, the resolution of $\phi_{\text {inst }}(x, y)+\phi_{\text {inst }}(-x,-y)$ is twice as coarse.

The primary disadvantage of the split arrangement is that the split between the mirror halves results in some loss of interferogram coverage, at the lowest angular frequencies. As a result, the reconstructed images are high-pass filtered, and have a different appearance from the direct images. A comparison of the imaging performance of the split arrangement and the current arrangement is shown in Fig. 12.

We determined that the improved resolution in in-
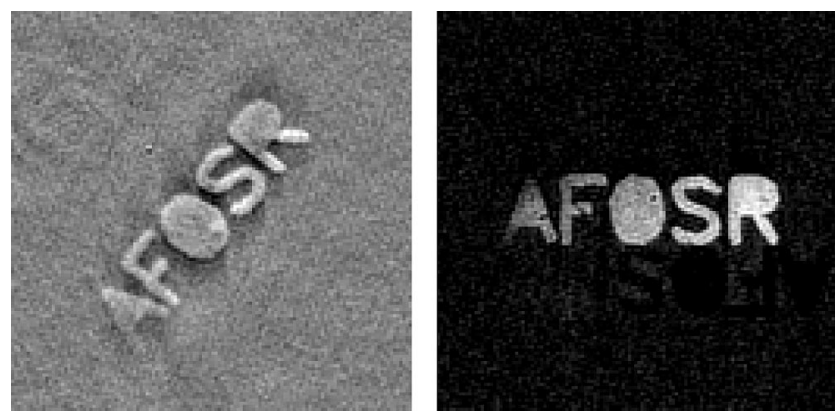

Fig. 12. Comparison of imaging performance under two QPI configurations. The left panel is the image produced by a split mirror QPI configuration, and the right panel is the image produced by the current QPI configuration. The split mirror configuration results in a loss of low-frequency information, producing a high-pass filtered image.

strumental phase adjustments from using a split mirror did not justify its increased complexity and the loss of $(u, v)$ coverage. However, further improvements in the optical design are certainly possible.

\section{References}

1. F. Roddier, "The effects of atmospheric turbulence in optical astronomy," in Progress in Optics, Vol. XIX, E. Wolf, ed. (North-Holland, 1981), pp. 281-376.

2. F. Roddier, Adaptive Optics in Astronomy (Cambridge University Press, 1999).

3. D. L. Fried, "Statistics of a geometric representation of wavefront distortion," J. Opt. Soc. Am. 55, 1427-1435 (1965).

4. P. Wizinowich, D. S. Acton, C. Shelton, P. Stomski, J. Gathright, K. Ho, W. Lupton, K. Tsubota, O. Lai, C. Max, J. Brase, J. An, K. Avicola, S. Olivier, D. Gavel, B. Macintosh, A. Ghez, and J. Larkin, "First light adaptive optics images from the Keck II telescope: a new era of high angular resolution imagery," Pub. Astron. Soc. Pacific 112, 315-319 (2000).

5. L. C. Roberts and C. R. Neyman, "Characterization of the AEOS adaptive optics system," Pub. Astron. Soc. Pacific 114, 1260-1266 (2002).

6. J. E. Baldwin, M. G. Beckett, R. C. Boysen, D. Burns, D. F. Buscher, G. C. Cox, C. A. Haniff, C. D. Mackay, N. S. Nightingale, J. Rogers, P. A. G. Scheuer, T. R. Scott, P. G. Tuthill, P. J. Warner, D. M. A. Wilson, and R. W. Wilson, "The first images from an optical aperture synthesis array: Mapping of Capella with COAST at two epochs," Astron. Astrophys. 306, L13-L16 (1996).

7. A. Labeyrie, "Attainment of diffraction limited resolution in large telescopes by Fourier analyzing speckle patterns in star images," Astron. Astrophys. 6, 85-87 (1970).

8. C. A. Haniff, C. D. Mackay, D. J. Titterington, D. Sivia, and J. E. Baldwin, "The first images from optical aperture synthesis," Nature 328, 694-696 (1987).

9. G. R. Ayers and J. C. Dainty, "Iterative blind deconvolution method and its applications," Opt. Lett. 13, 547-549 (1988).

10. J. B. Breckinridge, "Coherence interferometer and astronomical applications,” Appl. Opt. 11, 2996-2998 (1972).

11. C. Roddier and F. Roddier, "High angular resolution observations of Alpha Orionis with a rotation shearing interferometer," Astrophys. J. 270, L23-L26 (1983).

12. J. M. Mariotti, J. L. Monin, P. Ghez, C. Perrier, and A. Zadrozny, "Pupil plane interferometry in the near infrared I. Methodology of observation and first results," Astron. Astrophys. 255, 462-476 (1992). 
13. C. M. de Vos, J. D. Bregman, and U. J. Schwarz, "Pupil plane interferometry: Some conclusions from SCASIS," in Very High Angular Resolution Imaging, J. G. Robertson and W. J. Tango, eds. (Kluwer, 1994), pp. 419-420.

14. J. B. Breckinridge, "Two-dimensional white light coherence interferometer," Appl. Opt. 13, 2760-2762 (1974).

15. F. Roddier, C. Roddier, R. Petrov, R. Martin, G. Ricort, and C. Aime. "New observations of Alpha Orionis with a rotation shearing interferometer," Astrophys. J. 305, L77-L80 (1986).

16. K. Wallace, G. Hardy, and E. Serabyn. "Deep and stable interferometric nulling of broadband light with implications for observing planets around nearby stars," Nature 406, 700-702 (2000).

17. M. Born and E. Wolf, Principles of Optics (Cambridge University Press, 1999).

18. A. R. Thompson, Interferometry and Synthesis in Radio Astronomy (Wiley, 1986).

19. E. Ribak, C. Roddier, F. Roddier, and J. B. Breckinridge,
"Signal to noise limitations in white light holography," Appl. Opt. 27, 1183-1186 (1988).

20. K. T. Knox and B. J. Thompson, "Recovery of images from atmospherically degraded short-exposure photographs," Astrophys. J. 193, L45-L48 (1974).

21. N. Ageorges, J.-L. Monin, L. Desbat, and E. Tessier, "Phase and image reconstruction from interferometric imaging: Integration of the phasors," Astron. Astrophys., Suppl. Ser. 112, 163-171 (1995).

22. C. A. Hummel, J. T. Armstrong, A. Quirrenbach, D. F. Buscher, D. Mozurkewich, N. M. Elias, and R. E. Wilson, "Very high precision orbit of Capella by long baseline interferometry," Astron. J. 107, 1859-1867 (1994).

23. F. Roddier, "Interferometric imaging in optical astronomy." Phy. Rep. 170, 99-166 (1988).

24. B. D. Mason, W. I. Hartkopf, E. R. Holdenried, T. J. Rafferty, G. L. Wycoff, G. S. Hennessy, D. M. Hall, S. E. Urban, and T. E. Corbin, "Speckle interferometery at the US Naval Observatory VI," Astron. J. 120, 1120-1132 (2000). 